\title{
Persistent high prevalence of smoking reflects a major failure of public health
}

\author{
Sunitha cherukuri, \\ Browns college of pharmacy, Khammam, India.
}

\begin{abstract}
Corresponding Author: Sunitha cherukuri, Browns College of pharmacy, Khammam, India. E-mail: cherukuri.sunitha@gmail.com Received date: April 11,2018;Accepted date: May31,2019; Published date: June11, 2019.

Citation : Sunitha cherukuri, Persistent high prevalence of smoking reflects a major failure of public health, J. Addiction and AdolescentBehavior. Doi:10.31579/2688-7517/007
\end{abstract}

Copyright:(C2019Sunitha cherukuri. This isan open-access article distributed under theterms of The Creative Commons Attribution License, which permits unrestricted use, distribution, and reproduction in any medium, provided the original author and source are credited.

\begin{abstract}
Patients with mental health and addiction disorders are the only core cigarette smokers left in the Western world as the number of smokers in the general population has declined dramatically over the last decades. Simultaneously there is a gap in life expectancy of more than 20 years between patients who have been in treatment for mental disorders and the rest of the population and this gap has not declined in the last two decades. Adding to this are new research findings that show that cigarette smoking has a detrimental effect also on mental health being a risk factor for depressive symptoms.

There is therefore a need for concentrated action to curb the smoking epidemic among people with mental health and addiction disorders. There is limited research and guidelines on how to both prevent and cease smoking in this group. The aim of this paper is to give an overview of the current literature of the size of the problem, how to handle smoking cessation both individually and at system level and on the prevention of smoking.

The review confirms that the rate of smokers among patients with mental health and addiction disorders is huge with some figures pointing up to $80 \%$ in specific groups. It also confirms that cigarette smoking is a strongly addictive making the success rates low, especially without aides like snooze and e-cigarettes. It also shows, however, that it is safe to create a smoking free environment in all mental health and addiction units which also might be beneficial to the patients in the long run.
\end{abstract}

\section{Introduction}

Historically, smoking has been very common among mental health patients and mental healthcare workers, and only recently has smoking declined among healthcare professionals [1,2]. Smoking by mental health patients while receiving treatment for their mental health problems has been used to reduce feelings of aggression and anxiety [1]. The ability to smoke while in treatment has been used as a tool by healthcare providers to exact punishment or reward to influence the behavior of mental health patients. For many mental health professionals, smoking was seen as a way to break down barriers between themselves and their patients. Moreover, many mental health professionals had longstanding belief that mental health disorders lessened the detrimental effects of smoking [1].

Smoking remains very common among people with mental health problems, particularly those with substance abuse disorders [2]. When people with mental health disorders are receiving treatment, smoking poses a health risk to their fellow patients and the mental health professionals providing their care. Countries worldwide have prohibited smoking in the workplace in an effort to curb smoking and thereby protect the health of both the smokers and those in the vicinity of the smoker. Mental health treatment centers in many countries remain an important exception to this trend [1]. Given the high prevalence of smoking among patients receiving treatment, the clientele, staff, and healthcare providers at these treatment facilities are commonly exposed to second-hand smoke [2]. The aim of this narrative review is to present an overview of the prevalence of smoking among people with mental health disorders and the impact of
Smoking on mental health morbidities and life expectancy, and to describe efforts to curb smoking among people with mental health disorders, both in and out of treatment, and barriers to the enactment of prohibiting smoking at treatment sites. We conclude with proposals for future research and smoking policy updates.

\section{Smoking among People with Mental Health Disorders}

Tobacco use is still one of the leading preventable causes of death in the world killing up to half of its users [3]. The tobacco epidemic is one of the biggest public health threats, killing around six million people a year [3]. More than five million of those deaths are the result of direct tobacco use while more than 600,000 are the result of non-smokers being exposed to second-hand smoke [3]. The number of smokers in the general population is rapidly decreasing in the Western world. For example, in 1973 in Norway almost $45 \%$ of the populations were daily smokers, in 2004 this was reduced to $26 \%$, and last year only $13 \%$ of populations were daily smokers [4].

The severely mentally ill comprise a large proportion of the heavy smokers of today in the western world [2]. A third of all cigarettes smoked in England are the people with a mental disorder, and in contrast to the marked decline in smoking prevalence in the general population, smoking among those with mental disorders has changed little, if at all, over the past 20 years [5]. A study from the US based on the National Comorbidity Survey conducted from 1991 to 1992 reported that 44.3 percent of all cigarettes in America were consumed by individuals who live with mental illness and/or substance abuse disorders [6]. This means that persons with mental illness were about twice as likely to smoke as other persons, even at times when the general population smoking rate was higher

Impact of Smoking on Life Expectancy and Mental Health 
Life expectancy among mental health patients has recently become a pressing issue. Studies from the Nordic countries of Sweden, Finland, and Denmark show that men and women who have been in hospital with a psychiatric diagnosis, except for mental retardation and dementia, have a shortened life expectancy of 20 years and 15 years, respectively, compared to the rest of the population. Despite major changes in the health services available to people with mental illnesses, the life expectancy gap between people with a psychiatric hospitalization and the rest of the population has not changed significantly over the last 20 years [7].

The higher death rate is present in all psychiatric diagnostic groups, where it is highest for those with drug addiction and lowest for those with mood disorders [8]. About $40 \%$ of the total excess mortality among patients with schizophrenia is caused by external causes, primarily suicides, while approximately $60 \%$ can be attributed to somatic disease. For people with bipolar disorder or schizophrenia, the mortality rate when cardiovascular disease is present is 2-3 times higher than for the rest of the population [9].

Figures from Australia in the period between 1985 and 2005 show that the gap in life expectancy between those who have contact with mental health services and the rest of the population increased by 2.4 years for men and 1.6 years for women. The study showed further that $77.7 \%$ of the excess mortality was related to medical conditions, where cardiovascular diseases accounted for $29.9 \%$ and cancer for $13.5 \%$ excess mortality. Suicide was linked to only $13.9 \%$ of the reduction in life expectancy [10].

\section{Mental health}

In 2000, the US National Comorbidity Study found evidence that smoking preceded and was a risk factor for mental health problems [6]. Later studies confirmed this finding, such as Lineberry's observation that smoking is a risk factor for suicidality [11]. Similarly, a longitudinal study among teenagers showed that daily smokers at 15 years of age were more likely to be depressed 3 years later. This same study did not show that depressive symptoms increased smoking [12]. A large birth cohort study from Australia also identified an association between age at first tobacco use and subsequent psychosis-related outcomes in young adulthood [13]. All the studies mentioned are general-population based which exclude inpatient populations and thus make it impossible to study the impact of smoking among inpatients on their own health and on those around them.

In contrast to the evidence showing negative effects of smoking on mental health outcomes, a report by the Royal College of Psychiatrists summarizes the potential positive effects of cigarette smoking [5]. The investigators found that there is experimental evidence to suggest that nicotine can relieve symptoms of anxiety, depression, schizophrenia and attention deficit hyper activity disorder (ADHD). The authors also note, however, that nicotine withdrawal symptoms may then exacerbate symptoms of mental disorders. The evidence supporting a positive impact of smoking on mental health outcomes suggests that some people with mental disorders may self-medicate with nicotine to ameliorate mental health symptoms such as depression or anxiety. It is important to acknowledge these apparent positive effects when motivating patients for smoking cessation. On the other hand, it is important to state that the symptoms of mental disorders can be confused with or exacerbated by those of nicotine withdrawal, resulting in false attribution of cessation to the relief of symptoms of mental disorders [5].

Other studies have examined other relevant dimensions between smoking and mental health. One such study found that there was an association between smoking severity and poor self-reported quality of life connected to leisure activities, social relationships, and finances [14]. Another study showed that mental health patients who smoked had a poorer prognosis, more problems with medication, and more hospitalizations than non-smokers [15]. A recent meta-analysis of smoking and psychosis included 61 studies up to 2014 and involved 14,555 tobacco smokers and 273,162 non-smokers [16]. The investigators hypothesized that if the high rate of smoking among people with psychosis was related to self-medication, then smoking rates could be expected to be normal at the time of the first psychotic episode and subsequently increase in reaction to the symptoms. Instead, the analysis of case-control studies found that $57 \%$ of people with a first episode of schizophrenia were already smokers, for an overall odds ratio for smoking of 3.22 among people with an episode of schizophrenia (95\% confidence interval [CI], 1.63 - 6.33) [16]. The authors noted some evidence of publication bias. An additional sub-analysis of five longitudinal, prospective studies showed a more modest association, but daily smokers were still approximately twice as likely to develop new psychotic disorders as nonsmokers (relative risk, 2.18; 95\% CI, 1.23 - 3.85). In addition, those who smoked daily were found to develop psychotic illness approximately one year earlier than non-smokers. There was no significant difference in age of smoking debut between those who did and did not develop psychosis [16].

There have also been a few studies investigating the possible biological underpinnings of the association between psychosis and smoking. One study showed that nicotine could cause a change in the dopaminergic system through induction of super sensitivity of D2 receptors, which has been proposed as an explanatory mechanism for several risk factors for schizophrenia and as a common pathway for psychotic symptoms [17]. Finally, a key cluster of genes - CHRNA5, CHRNA3, and CHRNB5- on chromosome 15, which have been linked to schizophrenia in the largest genome-wide association study of the disease to date, are also associated with nicotine dependence and smoking behavior [18].

\section{Double Trouble - Smoking While In Mental Health Treatment}

Given the decline in smoking among mental healthcare workers, the potential advantage of closeness between mental health patients and their providers who smoke has diminished. Currently, smoking at treatment facilities must often take place in special shelters with specific ventilation systems, which removes some of the ease and comfort related to smoking [1]. Moreover, the combination of reduced interpersonal interaction related to smoking and ease of smoking may increase the patient's feelings of isolation and alienation. The stigma connected to smoking might also be a problem in creating social networks while in treatment and connecting with visiting family members. For smokers with mental health problems and substance abuse this creates a double marginalization where not only the mental health problems generate barriers to work and social interaction but smoking also introduces barriers to socialization.

\section{Efforts towards Smoking Cessation among People with Mental Health Disorders \\ Barriers to smoking cessation}

The US National Comorbidity Survey observed that more than a third of patients with a history of mental illness had quit smoking by the time of the survey [6]. Furthermore, they found that persons abstinent from alcohol had quit rates equal to those of persons without mental illness [6]. The Smoking and Mental health report states that smokers with mental disorders are just as likely to want to quit as those without, but are more likely to be heavily addicted to smoking [5].

So why then is it so difficult to reduce smoking among patients with mental health problems and especially those with severe problems? First, mental health care workers do not always view smoking as an addiction comparable to addiction to alcohol and other drugs. Rather, smoking is seen as one of few pleasures in an otherwise tumultuous life. Second, there is the belief among mental healthcare workers that pressure to quit might lead to feelings of shame and guilt among patients, of which they typically already have plenty. Moreover, the expectations of success with smoking cessation are extremely low [1].

Other obstacles to smoking cessation among patients with mental health problems come from experience-based knowledge among health workers. For example, the increased emotional stress they observe among patients abstinent from cigarettes. A strongly held belief is that to abandon smoking might lead to increased violent behavior. These are some of the reasons that health workers and managers may justify violating smoking laws. A third and more theoretical reason for allowing smoking is that cigarettes or smoking privileges are seen as a stable object while working on other addiction treatments [1]. 


\section{Quitting Measures}

There is increasing pressure from health workers to reduce smoking among patients to ensure a smoke free work environment. As discussed above, however, there are many barriers to attempts to "denormalize" smoking in treatment facilities. One important question is how to motivate people with mental health problems to reduce or quit smoking. General public information campaigns don't seem to work as smoking has not been reduced among people with mental health problems compared with the general population. An important measure that works especially well among youth is price control on cigarettes. While youth are sensitive to marked increases in the price of smoking, this measure seems to only increase the financial burden for those with mental health problems. Studies report some patients with mental health disorders spend up to $30 \%$ of their income on cigarettes. Since restrictions seem difficult to impose and public health measures are insufficient, innovative methods to reach people with mental health problems should be developed [5].

One approach may be to increase the impact of existing approaches, such as media campaigns, by tailoring them to the specific needs of the mental health patient populations. Also, given the opportunities presented by contacts with mental health services, these visits may be points at which to intervene to support smoking cessation and harm reduction. Smoke-free policies are more likely to be successful and effective if they are comprehensive, and can be implemented successfully in mental health settings with appropriate leadership and support strategies for patients and staff. Training and support to overcome prevailing misconceptions and negative or indifferent attitudes towards treating smoking among mental health staff is needed [3].

Psycho education for chronic psychiatric patients indicates that patients are willing to learn more about the effects of smoking, although most find it difficult to quit [19]. A review article mentioned that people with mental health disorders are as motivated to quit smoking as the general population, although those with psychotic disorders may be less motivated as individuals with depression [20]. A common experience however, is that health care providers are less motivated than their patients, which may be due to lacking of experience and tools to work with patients to reduce smoking. Moreover, health care workers at mental health treatment facilities are often working at capacity and introducing more interventions may be overwhelming and thus not implemented [1].

So, are there effective smoking cessation interventions? The combination of cognitive behavioral smoking cessation counseling session with cessation pharmacotherapy is effective in the general population, and has also been shown to be effective in people with mental disorders. Nicotine replacement therapy (NRT) \{nicotine patches, gums, sprays or lozenges $\}$ is effective in people with mental disorders, but may require high doses, for longer durations, and include more intensive behavioral support compared to the general population of smokers [21]. In addition to their effectiveness at reducing smoking among people with mental health problems, smoking cessation programs have also been shown to be highly cost-effective. A randomized controlled trail of a stage-based smoking cessation service at a psychiatric hospital found the intervention did not result in higher mental health care costs in the short-term and were highly costeffective over the projected lifetime of patients [21].

First line drugs like bupropion, varenicline and second line drugs nortriptyline and clonidine are effective in people with mental disorders, but should be used with appropriate supervision and monitoring [22]. Smoking cessation reduces the metabolism of some drugs, such as clozapine, necessitating prompt reduction in doses of affected drugs at the time of quitting, and increases in the event of relapse. Naltrexone hydrochloride is another drug that can be used for smoking cessation which reduces desire of smoking if combined with CBT (cognitive behavior therapy).Smokers who do not want to quit smoking, or else feel unable to make a quit attempt, should be encouraged to cut down on smoking, and to use snooze or e-cigarettes in line with the tobacco harm reduction guidance of the National Institute for Health and Clinical Excellence [23].

\section{Prevention}

There is a need for increased focus on the prevention of lifestyle diseases in people with mental illness based on the known recommendations on diet, smoking, alcohol, and exercise. Risk factors should be followed closely and interventions should be planned based on the individual patient's everyday life while aiming to create change with 'small steps'. There is good evidence that the prevention intervention of lifestyle diseases is effective among patients with mental disorders. Studies evaluating efforts to increase physical activity in people with schizophrenia are consistently positive [24]. Positive effects have also been observed among depressed patients, and these physical activity prevention interventions were also noted to be very cost effective [25].

Achieving cessation in $25 \%, 50 \%$, and $100 \%$ of people with mental disorders would, respectively, result in a gain of 5.5 million, 11 million, and 22 million undiscounted life-years in the UK [5]. At 3.5\% discounting, the corresponding figures are 1.4, 2.7, and 5.4 million life-years gained [5]. Harm reduction through lifelong substitution with medicinal nicotine is highly costeffective when compared with continuing smoking, at around $£ 8,000$ per quality-adjusted life-year (QALY) gained for lifetime nicotine patch use and $£ 3,600$ per QALY for inhalators. Addressing the high prevalence of smoking in people with mental disorders offers the potential to realize substantial cost savings as well as benefits in quantity and quality of life [5].

Child and adolescent mental health services, and local authority foster care and smoking policies, should explicitly protect children from passive smoke, and promote smoke-free foster homes. Professionals working with or caring for young people should provide positive non-smoking role models and be trained to deliver cessation advice and provide or arrange further support for those who want help to quit. In settings where young people are most vulnerable, such as adolescent in patient units, there should be a broad program of health promotion aimed at preventing initiation of smoking as well as smoking cessation [5].

\section{Conclusion}

People with mental health disorders smoke at higher rates, and they are more nicotine-dependent, and suffer greater morbidity and mortality from smoking-related illnesses than the general population. Helping people with mental health disorders to quit smoking is a public health priority; however, some mental health professionals assume that those with mental illness are not motivated to quit.

On the contrary, studies suggest that people with mental health disorders are indeed motivated to quit smoking, although more research is needed studying in-patient populations in particular [1]. The commonly held false belief that people with mental health disorders are not motivated to cease smoking means that opportunities to encourage smoking cessation among this disenfranchised group are being missed. It is likely that the persistent acceptance of smoking as normal behavior in primary and secondary care, and failure by health professionals to address smoking prevention as a health priority, drives and perpetuates the high prevalence of smoking among people with mental disorders.

This persistent high prevalence of smoking reflects a major failure of public health and clinical services to address the needs of a highly disadvantaged sector of society. There is a moral duty to address this problem in the future, and to prioritize the rights of people with mental disorders to the same protection and health interventions as the general population. Smoke-free policy is crucial to promoting smoking cessation in mental health settings. All healthcare settings used by people with mental disorders should be completely smoke free. 


\section{References}

1. Hirshbein LB. Smoking Privileges; Psychiatry, the Mentally Ill, and the Tobacco Industry in America. 2013 Rutgers University Press, New Jersey, USA.

2. Hall SM, Prochaska JD. Treatment of Smokers with CoOccurring Disorders: Emphasis on Integration in Mental Health and Addiction Treatment Settings. Annu Rev Clin Psychol. 2009;5:409-31. doi: 10.1146/annurev.clinpsy.032408.153614.

3. WHO. Fact sheet: Tobacco. 2015. Available from: http://www.who.int/mediacentre/factsheets/fs339/en.

4. Statistics Norway. Smoking Habits, 2014.2015.Available from: http://www.ssb.no/royk.

5. Lasser K, Boyd JW, Woolhandler S, Himmelstein DU, McCormick D, Bor DH. Smoking and mental illness: A population-based prevalence study. JAMA. 2000;284(20):2606-10.

6. Wahlbeck K, Westman J, Nordentoft M, Gissler M, Laursen TM. Outcomes of Nordic mental health systems: life expectancy of patients with mental disorders. $\mathrm{Br} \mathrm{J}$ Psychiatry. 2011;199(6):453-8. doi: 10.1192/bjp.bp.110.085100.

7. Nordentoft M, Wahlbeck K, Hällgren J, Westman J, Osby U, Alinaghizadeh $\mathrm{H}$, et al. Excess mortality, causes of death and life expectancy in 270,770 patients with recent onset of mental disorders in Denmark, Finland and Sweden. PLoS One. 2013;8(1):e55176. doi: 10.1371/journal.pone.0055176.

8. Laursen TM, Wahlbeck K, Hällgren J, Westman J, Ösby U, Alinaghizadeh $\mathrm{H}$, et al. Life expectancy and death by diseases of the circulatory system in patients with bipolar disorder or schizophrenia in the Nordic countries. PLoS One. 2013;8(6):e67133. doi: 10.1371/journal.pone.0067133.

9. Lawrence D, Hancock KJ, Kisely S. The gap in life expectancy from preventable physical illness in psychiatric patients in Western Australia: retrospective analysis of population based registers. BMJ. 2013;346:f2539. doi: 10.1136/bmj.f2539.

10. Lineberry TW, Allen JD, Nash J, Galardy CW. Populationbased prevalence of smoking in psychiatric inpatients: a focus on acute suicide risk and major diagnostic groups. Compr Psychiatry. 2009;50(6):526-32.

doi: 10.1016/j.comppsych.2009.01.004.

11. Lien L, Sagatun A, Heyerdahl S, Sogaard AJ, Bjertness E. Is the relationship between smoking and mental health influenced by other unhealthy lifestyle factors? Results from a 3-year followup study among adolescents in Oslo, Norway J Adolesc Health. 2009;45(6):609-17. doi: 10.1016/j.jadohealth.2009.04.011.

12. McGrath JJ, Alati R, Clavarino A, Williams GM, Bor W, Najman JM, et al. Age at first tobacco use and subsequent psychosis-related outcomes: A birth cohort study. Aust N Z J Psychiatry. 2015. pii: 0004867415587341.
13. Dixon L, Medoff DR, Wohlheiter K, DiClemente C, Goldberg R, Kreyenbuhl J, et al. Correlates of severity of smoking among persons with severe mental illness. Am J Addict. 2007;16(2):101-10.

14. Morris CD, Waxmonsky JA, May MG, Tinkelman DG, Dickinson M, Giese AA. Smoking reduction for persons with mental illnesses: 6-month results from community-based interventions. Community Ment Health J. 2011;47(6):694-702. doi: 10.1007/s10597-011-9411$\mathrm{z}$.

15. Gurillo P, Jauhar S, Muray RM, MacCabe JH. Does tobacco use cause psychosis? Systematic review and meta-analysis. Lancet Psychiatry. 2015;2(8):718-25. doi: 10.1016/S2215-0366(15)001522.

16. Novak G, Seeman P, Le Foll B. Exposure to nicotine produces an increase in dopamine D2(High) receptors: a possible mechanism for dopamine hypersensitivity. Int J Neurosci. 2010;120(11):691-7. doi: $10.3109 / 00207454.2010 .513462$.

17. Schizophrenia Working Group of the Psychiatric Genomics Consortium. Biological insights from 108 schizophrenia-associated genetic loci. Nature. 2014;511(7510):421-7. doi: 10.1038/nature13595.

18. Hitsman B, Moss TG, Montoya ID, George TP. Treatment of tobacco dependence in mental health and addictive disorders. Can J Psychiatry. 2009;54(6):368-78.

19. Siru R, Hulse GK, Tait RJ. Assessing motivation to quit smoking in people with mental illness: a review. Addiction. 2009;104(5):719-33. doi: 10.1111/j.1360-0443.2009.02545.x.

20. Barnett PG, Wong W, Jeffers A, Hall SM, Prochaska JJ. Costeffectiveness of smoking cessation treatment initiated during psychiatric hospitalization: analysis from a randomized, controlled trial. J Clin Psychiatry. 2015;76(10):e1285-91. doi: 10.4088/JCP.14m09016.

21. Tidey JW, Miller ME. Smoking cessation and reduction in people with chronic mental illness. BMJ. 2015;351:h4065. doi: 10.1136/bmj.h4065.

22. National Institute for Health and Care Excellence. Smoking: tobacco harm-reduction approaches overview. Available from: http://pathways.nice.org.uk/pathways/smoking-tobaccoharm-reduction-approaches.

23. Gorczynski P, Faulkner G. Exercise therapy for schizophrenia. Cochrane Database Syst Rev. 2010;(5):CD004412. doi: 10.1002/14651858.CD004412.pub2.

24. Park AL, McDaid D, Weiser P, Von Gottberg C, Becker T, Kilian R. Examining the cost effectiveness of interventions to promote the physical health of people with mental health problems: a systematic review. BMC Public Health. 2013;13:787. doi: 10.1186/1471-245813-787. 